Annuaire suisse de politique de développement

$14 \mid 1995$

Annuaire Suisse - Tiers Monde 1995

\title{
Réflexions sur le thème de la cohérence
}

\section{Peter Maurer}

\section{OpenEdition}

\section{Journals}

Édition électronique

URL : http://journals.openedition.org/aspd/1445

DOI : 10.4000 /aspd. 1445

ISSN : 1663-9669

\section{Éditeur}

Institut de hautes études internationales et du développement

\section{Édition imprimée}

Date de publication : 1 février 1995

Pagination : 160-164

ISSN : 1660-5934

\section{Référence électronique}

Peter Maurer, «Réflexions sur le thème de la cohérence », Annuaire suisse de politique de

développement [En ligne], 14 | 1995, mis en ligne le 03 mai 2013, consulté le 08 septembre 2020. URL http://journals.openedition.org/aspd/1445 ; DOI : https://doi.org/10.4000/aspd.1445 


\title{
Réflexions sur le thème de la cohérence
}

\author{
Peter Maurer, Direction politique, DFAE
}

La cohérence comme thème du rapport sur la politique extérieure dans les années 90

Dans son Rapport sur la politique extérieure de la Suisse dans les années 90 , le Conseil fédéral souligne que la cohérence en matière de politique extérieure est importante à trois niveaux:

- maintenir l'attrait politique et économique de la Suisse à l'échelle internationale;

- assurer un engagement aussi efficace que possible de moyens limités;

- défendre de manière optimale nos intérêts à l'échelle internationale par une présence homogène.

Une procédure adéquate doit permettre de répondre à ces besoins de cohérence. Ailleurs, il est dit que le but du projet présenté est de montrer que "la politique extérieure d'un pays constitue un tout". Que pourrait être ce tout si ce n'était un tout cohérent? Et à quoi ressemble ce tout? 
Le Rapport établit les objectifs en matière de politique extérieure sous la forme d'un pentagone. II n'existe d'ailleurs pas de hiérarchie entre les différents objectifs cités ci-dessous:

- le maintien et la promotion de la sécurité et de la paix,

- l'engagement en faveur des droits de l'homme, de la démocratie et des principes de l'Etat de droit,

- l'accroissement de la prospérité commune,

- la promotion de la cohésion sociale,

- la préservation du milieu naturel.

Toutes les activités déployées par rapport à ces cinq objectifs doivent, au demeurant, être en adéquation avec une politique d'intérêts globalement comprise. La sauvegarde des intérêts est "avant tout la fonction première et universelle, en un certain sens la raison d'être et le mobile inhérent de toute politique extérieure". Cela signifie que, outre les intérêts économiques, il faut également définir - entre autres - quels sont les intérêts sur le plan de la politique de sécurité et d'environnement, sans oublier la sauvegarde durable de ces intérêts.

Les conflits entre les objectifs, donc les problèmes de cohérence, sont intégrés dans un tel concept de politique extérieure qu'on ne peut en faire abstraction. Dans ce cadre une problématique surgit: par rapport à une utilisation efficace des ressources de l'Etat, à une représentation aussi convaincante que possible vers l'extérieur et à l'explication de la politique extérieure à l'opinion publique, il importe que la cohérence atteigne un degré élevé. Du fait du grand nombre d'intérêts divergents au sein d'une société pluraliste, une cohérence excessive n'est pas souhaitable, car elle traduit une hiérarchie des priorités qui relègue tel ou tel intérêt derrière les autres.

Du fait de cette exigence d'une politique extérieure cohérente, nos relations extérieures ne peuvent pas être entièrement exemptes de contradictions. C'est plutôt la question suivante qui devient centrale: comment pouvons-nous obtenir les meilleurs résultats dans le cadre des cinq objectifs précités? Comment et par quelles procédures règle-t-on politiquement les conflits entre objectifs?

Deux stratégies se présentent en premier lieu, à savoir:

- l'optimisation de l'utilité (par quelles mesures prises dans un domaine donné peut-on obtenir le maximum d'effets positifs dans un autre domaine?)

- la minimisation des coûts (quelles sont les mesures prises dans un domaine qui ont le minimum d'effets négatifs dans les autres?)

\section{Les différents niveaux de la cohérence}

La notion de cohérence en matière de politique extérieure comporte de nombreuses facettes et doit prendre en compte les problèmes réels, la réthorique politique, les souhaits et les courants du moment. 
Des questions se posent à différents niveaux et il est difficile de trancher pour savoir si une décision doit être prise plutôt en faveur de la cohérence ou en faveur d'intérêts peut-être tout aussi légitimes. A ce propos, voici en résumé quelques points de réflexion:

\section{La cohérence dans le temps}

Comment se situe une décision par rapport à des décisions comparables qui l'ont précédée? Y a-t-il une continuité par rapport à des décisions prises auparavant et, par conséquent, une cohérence ou bien est-ce une discontinuité qui entraîne donc une incohérence? Dans quelles conditions peut-on et doit-on renoncer à ce qui a été jusqu'ici la pratique?

Exemple: pendant de longues années, la Suisse a toujours cessé d'autoriser des exportations de matériel de guerre à un pays donné, mais elle les interdit dorénavant.

Cohérence entre les affaires intérieures et les affaires extérieures

A-t-on les mêmes critères ou tout au moins les mêmes orientations ou les mêmes impulsions politiques pour les affaires intérieures et les affaires extérieures?

Exemple: la Suisse connaît elle-même sur son territoire de sévères prescriptions en matière de protection de l'environnement, mais apporte son soutien dans un autre pays, au moyen d'un crédit mixte, à la construction d'une usine qui nuit considérablement à l'environnement et qui n'obtiendrait pas d'autorisation d'exploitation dans notre pays. Ou encore: la Suisse s'engage pour des règles d'accès au marché aussi libérales que possible, mais dispense de cette exigence certains secteurs de sa propre économie.

Cohérence avec la politique extérieure d'autres pays

Dans quelles circonstances est-il sensé d'adopter des mesures politiques de la communauté internationale, "de l'UE", "de l'Europe en général», "d'un groupe d'Etats industriels" et dans quelles circonstances faut-il prendre des mesures différentes?

Exemple: la majorité des pays industriels se mettent d'accord sur une harmonisation de leurs prescriptions en matière de contrôle des exportations. Dans quelle mesure les dispositions spéciales prises par la Suisse dans ce domaine sont-elles sensées et réalistes? La majorité des pays d'Europe occidentale fournit du matériel de guerre à un pays donné qui, au sens strict de la législation suisse, est à considérer comme une zone de tension. Quel sens peut avoir le fait que la Suisse applique une procédure d'autorisation plus sévère?

Cohérence entre les objectifs et les moyens

Existe-il une corrélation raisonnable entre les objectifs de politique extérieure et les moyens (en hommes et en ressources financières) utilisés à cette fin?

Exemple: la Suisse explique que l'atténuation des antagonismes Nord-Sud représentait une priorité de sa politique extérieure, mais réduit pour des raisons 
de restrictions budgétaires les crédits accordés à cette activité. Le soutien des pays d'Europe de l'Est et d'Europe centrale est désigné comme une priorité de la politique européenne de la Suisse, mais cela n'empêche pas, là aussi, une réduction des crédits.

\section{Cohérence entre les différents acteurs}

Jusqu'à quel point existe-t-il une cohérence entre les acteurs de l'Etat et les acteurs du secteur privé?

Exemple: vu les tensions qui caractérisent les relations bilatérales avec un pays, la Suisse refuse de donner son accord à un rééchelonnement plus favorable de sa dette extérieure; dans le même temps, un groupe d'entreprises privées dirigées par des responsables suisses conclut un contrat pour une commande se montant à plusieurs milliards.

\section{La cohérence par la procédure}

La cohérence en matière de politique extérieure ne peut être améliorée - pour autant qu'elle soit considérée comme une condition politique souhaitable qu'au moyen d'une procédure ad hoc. Ce point appelle la remarque suivante:

- le système de collégialité et la conception de sa procédure au niveau administratif (rapports entre les départements) offrent sur le plan formel la plate-forme idéale d'une politique cohérente. Les structures de décision actuelles garantissent que la quasi-totalité des principales décisions puissent être discutées et adoptées à l'écheion gouvernemental. En Suisse, l'ampleur de la cohérence est ainsi tout à fait directement une fonction de la volonté politique d'être cohérent.

- Si, dans cette procédure, des carences apparaissent, celles-ci peuvent être éliminées dans une large mesure grâce à des mesures d'organisation à l'échelon du département. Déterminer le nombre de personnes qui doivent être chargées au sein d'un département de contrôler la cohérence dans l'élaboration des décisions du Conseil fédéral en matière de politique extérieure est en fin de compte une simple question d'utilisation des ressources. L'information exhaustive et fournie dans les délais au DFAE concernant toutes les activités de politique extérieure significatives constitue au demeurant la base de tous les efforts déployés pour réaliser au niveau administratif une politique extérieure cohérente.

- En outre, les chances de pratiquer une politique extérieure aussi cohérente que possible sont les plus grandes dans les domaines où sont définis des mécanismes de consultation juridiquement valables, comme c'est par exemple le cas de la loi sur le matériel de guerre qui garantit une prise en compte en temps utile des différents points de vue. Ces chances sont d'autant plus restreintes si l'on prévoit uniquement des consultations entre divers offices et départements.

- Dans cette perspective, cela vaudrait la peine de vérifier (à l'instar des clauses générales prévues dans l'ordonnance réglant les tâches liées au 
contrôle des demandes de crédit et d'autres requêtes par l'administration financière, c'est-à-dire par le conseil et l'instruction juridique du Département fédéral de justice et police), s'il ne faudrait pas prévoir un contrôle de la compatibilité et un contrôle de la cohérence en matière de politique extérieure plus rigoureux de la part du DFAE. Cela pourrait par exemple signifier que chaque projet revêtant une importance sur le plan de la politique extérieure présenté au Conseil fédéral devrait être assorti d'une prise de position quant à sa cohérence en matière de politique extérieure.

En ce qui concerne la cohérence qui pourrait être réalisée selon les procédures suivies jusqu'ici ou, le cas échéant, encore améliorées, une évidence s'impose aujourd'hui: de simples denial policies, autrement dit des interdictions décidées au nom de la cohérence ne sont valables que dans des cas exceptionnels, par exemple lorsque la Suisse adhère aux sanctions de la communauté des Etats contre un pays qui viole le droit ou lorsque du matériel de guerre risque d'être livré dans des zones où règnent visiblement des tensions.

On devrait rechercher beaucoup plus souvent des "mesures positives". Un dialogue entre les acteurs en Suisse qui défendent des intérêts différents devrait offrir à moyen et à long terme la meilleure garantie pour trouver un train de mesure qui sera mieux accepté sur le plan intérieur et plus cohérent sur le plan de la politique extérieure. Faute de quoi nous devrons sans cesse nous reposer cette question: pourquoi reste-t-il, malgré toutes les procédures et les contrôles de cohérence, autant d'incohérence? 